\title{
Aseptic loosening of femoral components - materials engineering and design considerations
}

\begin{abstract}
Aseptic loosening is one of the main reasons for the revision of a total knee replacement (TKR). The design of the key component of a TKR, the femoral component, is particularly problematic because its failure can be the result of different causes. This makes the development of new biomaterials for use in the femoral component a challenging task. This paper focuses on the engineering design aspects in order to understand the limitations of current materials and design deficiencies. The paper describes the introduction of a new biomaterial for a femoral component and justifies the recommendation to use multifunctional materials as a possible solution to aseptic loosening. The potential advantages of applying functionally graded biomaterials (FGBMs) in prosthetic femur are explained by reducing the leading causes of failure including wear, micro-motion and stress-shielding effect. The ideas presented in this paper can be used as the basis for further research on the feasibility and advantages of applying FGBM in other superior implant designs.
\end{abstract}

Keyword: Knee prosthesis; Biomaterials; Functionally graded materials; Composites; Selection for material properties; Engineering design 